\title{
Demokratische Wege
}




\section{Demokratische Wege}

Deutsche Lebensläufe aus fünf Jahrhunderten

Herausgegeben von

Manfred Asendorf und Rolf von Bockel

Verlag J.B. Metzler

Stuttgart - Weimar 
Projekt- und Redaktionsleitung: Manfred Asendorf

Redaktion: Rolf von Bockel

Korrekturarbeiten: Brigitte Domurath, Elfie Jehle, Jutta Kaiser, Sabine Matthes

Textverarbeitung: Meike Tadsen, Jutta Wolff, Diana Wolter

Register: Dieter Fuchs

Bildbeschaffung: Katherina Wilts

Wissenschaftliche Fachberatung: Helmut Bleiber, Elisabeth Böhmer, Klaus Briegleb, Hans-Werner Engels, Jens Flemming, Walter Grab, Franklin Kopitzsch, Wolfgang Kraushaar, Hartmut Ludwig, Friedrich Wilhelm Marquardt, Julius H. Schoeps, Winfried Steffani, Klaus Thomamüller, Ulla Wischermann

Die Deutsche Bibliothek - CIP-Einheitsaufnahme

Demokratische Wege : deutsche Lebensläufe aus fünf Jahrhunderten / hrsg. von Manfred Asendorf und Rolf von

Bockel. - Stuttgart ; Weimar : Metzler, 1997

ISBN 978-3-476-01244-9

NE: Asendorf, Manfred [Hrsg.]

ISBN 978-3-476-01244-9

ISBN 978-3-476-03551-6 (eBook)

DOI 10.1007/978-3-476-03551-6

Dieses Werk einschließlich aller seiner Teile ist urheberrechtlich geschützt. Jede Verwertung außerhalb der engen Grenzen des Urheberrechtsgesetzes ist ohne Zustimmung des Verlages unzulässig und strafbar. Das gilt insbesondere für Vervielfältigungen, Übersetzungen, Mikroverfilmungen und die Einspeicherung und Verarbeitung in elektronischen Systemen.

(C) 1997 Springer-Verlag GmbH Deutschland

Ursprünglich erschienen bei J.B. Metzlersche Verlagsbuchhandlung und Carl Ernst Poeschel Verlag GmbH in Stuttgart 1997 


\section{Inhalt}

Vorwort VII

Lexikon 1

Alphabetisches Verzeichnis der Biographien 717

Chronologisches Verzeichnis nach Geburtsjahren 722

Verzeichnis der Autorinnen und Autoren 727

Namenregister 730

Bildquellen 747 


\section{Vorwort}

Eine Volksversammlung, die durch Abstimmung die Regierung nötigt, einer Gruppe junger Ausländerinnen Asyl zu gewähren, obwohl damit die Gefahr außenpolitischer Verwicklungen heraufbeschworen wird - dieses Szenario entstammt nicht einer Chronik unserer Zeit, es bildet den Kern der ältesten bekannten literarischen Auseinandersetzung mit der Demokratie, der Tragödie des Aischylos Die Schutzflehenden, aufgeführt in Athen wahrscheinlich im Jahre 463 v. Chr.. Das Wort "Demokratie ist zuerst bei Herodot Mitte des 5. Jahrhunderts v.Chr. bezeugt

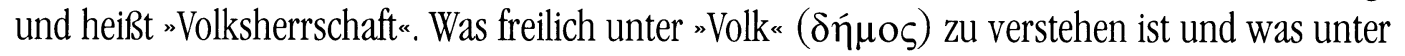

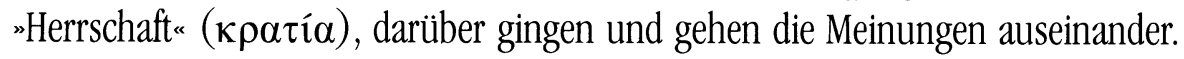

"Demos" bedeutet ursprünglich "alle« oder "viele«: gemeint waren relativ wenige: diejenigen, die als politisch berechtigte Staatsbürger in der Mehrheit waren, jedoch der Masse der Bevölkerung, den Sklaven (rechtlich als Sache behandelt), Frauen und Fremden gegenüber, eine kleine Minderheit darstellten. Wo Alexis de Tocqueville in seinem berühmten Buch Über die Demokratie in Amerika (1835/1840) vom "allgemeinen Wahlrecht» spricht, muß sich der Leser vergegenwärtigen, daß hier von nur 5 Prozent der Bevölkerung in den Vereinigten Staaten die Rede ist: Im Amerika des Tocqueville-Buches waren die Indianer, die Farbigen, die Frauen und ein großer Teil der Weißen vom Wahlrecht ausgeschlossen. Was die Frauen angeht, so hat es auch in Westeuropa bis ins 20. Jahrhundert gedauert, bis sie das Wahlrecht erlangten. Selbst neuere revolutionsgeschichtliche Darstellungen erwecken den Eindruck, es verstehe sich von selbst, daß die später so genannten »revolutionären Demokraten «, etwa 1793, zur Zeit der Französischen Revolution, oder in Deutschland 1848/49, in der Regel vom Frauenwahlrecht nichts hielten - von weitergehender politischer Partizipation zu geschweigen. Insofern ist der "mainstream" der Demokratiegeschichte auf weite Strecken ein "male stream" (Mary O'Brien).

"Kratia" bedeutet Macht, Gewalt, Herrschaft: Herrschaft ist nach Max Weber in dem allgemeinsten Sinne "Machtausübung, also die Möglichkeit, den eigenen Willen dem Verhalten anderer aufzuzwingen «. Die Durchsetzung des allgemeinen und gleichen Wahlrechts vorausgesetzt, müssen wir uns gleichwohl fragen, wie sich Demokratie konkret verwirklicht, zumal doch offenkundig ist, daß nicht alle gleichzeitig und in gleicher Weise herrschen können. Weitere Fragen stellen sich, wenn man der Auffassung folgt, daß Demokratie den Umfang der Befehlsgewalt minimiert oder wenigstens minimieren sollte - möglichst gegen Null. Und noch komplizierter wird es, wenn wir Demokratie als ein Prinzip begreifen, eine dynamische Kraft, die tendenziell, wenn auch keineswegs providentiell, wie Tocqueville glaubte, alle Bereiche der Gesellschaft durchdringt. Demokratie ist freilich nicht einfach, um ein beliebtes soziologisches Wort zu benutzen, ein "Projekt». Eher ist sie eine Lebensform, fragil und gefährdet. Und, wie Heinrich von Treitschke, ein Zeitgenosse des 19. Jahrhunderts und Gegner der Demokratie, richtig erkannt hat, eine "Contradictio in adjecto*. 
Schon die Staatslehrer der griechischen Antike wußten, um was es sich handelt. Seit Aristoteles (4. Jahrhundert v.Chr.) sind grundlegende Erkenntnisse benannt, die bis heute zum eisernen Bestand aller Demokratietheorien gehören: Wesentliche Merkmale der Demokratie sind Freiheit und Gleichheit. Freiheit und Gleichheit sind nicht identisch, aber auch nicht unaufhebbare Gegensätze. Der Gedanke der Freiheit verlangt, daß "das Regieren und Regiertwerden reihum geht" und zugleich, daß "jeder Staatsbürger mit dem anderen gleiches Recht haben soll«. Das demokratische Recht ist die Gleichheit nach der Zahl. Wo dies für Recht gilt, beschließt die Mehrzahl. "Daraus folgt aber, daß in den Demokratien die Armen größere Gewalt besitzen als die Reichen, denn sie bilden die Mehrzahl«. Und an anderer Stelle heißt es bei Aristoteles mit pessimistischem Unterton: "Denn immer sind es die Schwächeren, die nach Recht und Gleichheit suchen, die Stärkeren aber kümmern sich nicht darum». Eine weitere Grundbestimmung der Demokratie ist daher, "leben zu können, wie man will«, oder, wie wir heute sagen würden, selbstbestimmt.

Viele Zeitgenossen des 19. und 20. Jahrhunderts, darunter bedeutende Gelehrte unterschiedlichster Herkunft, stellten und stellen sich die Sache so vor, als ob mit der Französischen Revolution von 1789 der Vorhang aufging - und auf der Bühne erscheint "die moderne Demokratie“. Demgegenüber hat der Althistoriker (und Marxist) Arthur Rosenberg (1889-1943) gezeigt, in welch hohem Maß sich die Republikaner des 18. Jahrhunderts, vor allem in Frankreich und in Nordamerika, im Denken und Handeln dem Erbe der Antike verpflichtet fühlten. Wenn der antike Name der Demokratie in der Neuzeit seine Auferstehung habe feiern können, so sei das letztlich auf die "objektive Übereinstimmung der gesellschaftlichen Tatsachen" zurückzuführen, welche die Ähnlichkeit zwischen den modernen revolutionären Bewegungen und denen der Antike schuf. Noch weiter ging der liberale Aristokrat Tocqueville, indem er das demokratische Prinzip aus dem begrenzten revolutionsgeschichtlichen Zusammenhang löste und als "allmähliche Entwicklung zur Gleichheit der Bedingungen" ("égalité des conditions") beschrieb, die seit vielen Jahrhunderten über alle Hindernisse hinweg voranschreite.

Mit dieser Feststellung verband Tocqueville - zu einer Zeit, als Monarchisten, Adlige und große Teile des Bürgertums Demokratie auf Anarchie zu reimen pflegten - die Prognose, daß einmal etablierte Demokratien einen konservativen Charakter annähmen.

Damit dürfte zusammenhängen, daß in unserer Zeit, am Ende des 20. Jahrhunderts, mancher Beobachter die Debatte um die Wertsetzungen von 1789 bereits für verbraucht und für beendet erklärte. Der Zusammenbruch des "realexistierenden“, von kommunistischen Regimen verwalteten Sozialismus, zufällig in das Bicentenaire, das 200. Geburtsjahr der Französischen Revolution fallend, hat allenthalben die "demokratische Frage" wiederbelebt. Auch die akademische Intelligenz, jahrzehntelang eher desinteressiert, ist neugierig geworden. Unverstellt durch systembedingte legitimatorische Zwecke und Absichten an der Frontlinie zwischen "links" und "rechts", "fortschrittlich" und "reaktionär" ist jetzt die innere, allen ideologischen Selbstbestätigungen (häufig anmutend wie das Pfeifen im dunklen Walde) vorgängige, 
gesellschaftliche Sprengkraft der Demokratieproblematik (wieder) sichtbar. Gescheitert sind Versuche, die spätestens seit den westeuropäischen Revolutionen von 1848 immer größer gewordene Kluft zwischen Arbeiterbewegungssozialismus und bürgerlich-liberalem Verfassungsstaat mit nominalistischer Kampfrethorik auszufüllen oder zu verdecken. Dies gilt, wenn auch in unterschiedlicher Weise und Bewertung für die Identifikation von politbürokratischer Diktatur mit "demokratischem Zentralismus" wie für die dem entgegengesetzte Formel vom "demokratischen Sozialismus" und für die Beschwörung von "freiheitlich-demokratischer Grundordnung « zur Kennzeichnung einer rechtsstaatlich und staatsrechtlich definierten Bürgerbeteiligung, die durch Verfahren und Institutionen kanalisiert ist.

Die in dem Begriff Demokratie sprachlich und politisch eingebundene Spannung zwischen Gleichheit und Freiheit sowie die beide verklammernde Solidarität ("Brüderlichkeit«) ist aufgebrochen. Die Frontlinie, so zeigt sich, verläuft nicht nur zwischen "Demokraten" und Gegnern der Demokratie, sondern ist der demokratischen Wirklichkeit selbst zu eigen. Und sie ist es, seit es demokratische Bewegungen in der Geschichte gibt. Die demokratische Frage sah und sieht sich daher mit einer doppelten Schwierigkeit konfrontiert: mit dem Umstand, daß, nach aller geschichtlichen Erfahrung, die Entwicklung zur Gleichheit der Bedingungen, d.h. zugleich zu immer mehr Planung, Bürokratie und Reglementierung, die Autonomie des Einzelnen einebnet, während doch umgekehrt ohne ein erhebliches Maß an Gleichheit Freiheit im Sinne von Selbstbestimmung nicht möglich ist. Die Paradoxie wird schon im Wortgebrauch deutlich: Denn wann und wo wäre dieses Maß erreicht, wenn gesellschaftliche Gleichheit als Endzustand gedacht wird? Und wo Freiheit, wenn Freiheit, ebenfalls seinem radikalen Sinn entsprechend, den Gegensatz dazu bedeutet?

Von Demokratie und Demokratietheorie war die Rede. Indes: "Es sind nicht die Artikel einer Konstitution, die eine Demokratie ausmachen“, meinte der 1988 gestorbene französische Liberalismus- und Demokratieforscher Georges Burdeau. Und er fügte hinzu: "Es gibt nicht die Demokratie an sich, es gibt nur Demokraten «. Daß Demokratien oder demokratische Verhältnisse Menschen mit demokratischer Gesinnung erfordern (freilich auch hervorbringen), ist bekannt. Kaum ein bedeutender politischer Denker, der das nicht betont hätte, freilich in der Regel, um sich dann desto unbefangener generalisierenden politischen Ideen, theoretischen Systemen und determinierenden anonymen Kräften zuzuwenden. Ein Sammelsurium von Vorurteilen über den "Demokraten" schleppte sich auf diese Weise durch die Jahrhunderte, deren Ausgangspunkt in dem herben Verdikt Platons (5./4. Jahrhundert v.Chr.) liegt: "Es ist keine Ordnung und keine Notwendigkeit in seinem Leben «. Demokraten als unsichere Kantonisten, Demagogen, Feinde der Ordnung, intellektuell, charakterlich und politisch "Mittelmaß" verkörpernd. Andererseits wurde mit Rousseau (1712-1778) die Meßlatte so hoch gelegt, daß sie von niemandem übersprungen werden kann: "Wenn es ein Volk von Göttern gäbe, würde es sich demokratisch regieren«. Und: "Eine so vollkommene Regierung paßt für Menschen nicht«.

Das vorliegende Werk handelt von Menschen, von denkenden und fühlenden, von handelnden 
und leidenden, gewiß auch von irrenden Menschen, auch von Menschen, die selbst Schuld auf sich luden. Wer wie der deutsche Jakobiner Eulogius Schneider Anno 1793 mit einer fahrbaren Guillotine durch die Lande zieht und von Amts wegen Todesurteile verhängt, mag ein glühender Freund der Gleichheit sein - er übersieht nur, daß der gewaltsam zugefügte Tod neben der Folter und der Knechtschaft die extremste Demonstration und Form der Ungleichheit darstellt.

Dieses Werk ist ein biographisches Lexikon zur deutschen Demokratiegeschichte. Die Herausgeber haben sich bei der Konzeption von folgenden Grundüberlegungen leiten lassen:

Erstens: Da der Begriff der Demokratie - wie unter prinzipiellen Gesichtspunkten einleitend bereits erörtert - weder vor noch während seiner politischen Institutionalisierung und sozialen Ausgestaltung - identisch mit seiner realen Geschichte war und ist, ist es möglich, aber auch nötig, einen vergleichsweise langen Zeitraum ins Auge zu fassen: vom Beginn des 16 . Jahrhunderts bis 1945 (stellenweise noch darüber hinaus). Die österreichische Geschichte ist bis zum preußisch-österreichischen Krieg 1866 bzw. bis zur Gründung des Norddeutschen Bundes (ein Jahr später als Vorstufe zum Deutschen Reich) mitberücksichtigt. Reformation und Bauernkrieg zum Ausgangspunkt zu wählen, bedarf kaum der Erläuterung, werden diese Ereignisse doch in der Geschichtswissenschaft dem Beginn der "Neuzeit" oder der "Moderne" zugerechnet, als mit der beschleunigten Ausbreitung der Textreproduktion mit beweglichen Lettern, des Buchdrucks, ein wichtiges Moment demokratischen Handelns geschaffen wurde. Daß Reformation und Bauernkrieg im übrigen auch heute noch politische Energien freizusetzen vermögen, zeigt eine Meldung, die im September 1996 durch die Presse ging. Danach hat der Leipziger Kirchenhistoriker Kurt Nowak auf einem europäischen Theologenkongreß vor dem Hintergrund jüngster deutscher Geschichte "Demokratieblockaden« bei Ost- wie Westdeutschen, insbesondere bei evangelischen Christen, beklagt und gefordert, "das Erbe der Reformation unter dem Demokratiegesichtspunkt neu zu erschließen".

Zweitens: Wenn richtig ist, was Goethe in Wilhelm Meisters Lehrjabre sagt, daß der Geist, aus dem wir handeln, das Höchste ist und durch Worte allein nicht deutlich wird, dann ist der Versuch, durch Lebensläufe die demokratische Frage zu beleuchten, besonders gut geeignet. Denn hier verschränken sich Worte und Taten im Medium der Mentaliät, treffen sich soziale Figurationen mit individuellen Bedürfnissen.

Das Lexikon ist kein "Museum der Demokraten«. Die Herausgeber schreiben kein DemokratieMuster vor, sie wollen insofern weder eigene Überzeugungen bestätigt finden noch "pädagogisch « überzeugen. Sie haben Biographien gesammelt, um im Spannungsfeld von Gleichheit und Freiheit die Verwerfungen und Brüche festzumachen, die zu Aufstieg und Fall, zu Konkretion und Perversion des demokratischen Gedanken, zu den wiederholten Take-Offs der Demokratie und zu ihren Bruchlandungen oder gar Katastrophen geführt haben.

Das Buch enthält 420 Biographien, rund 200 namhafte Historiker, Politik- und Literaturwissenschaftler haben als Autoren mitgewirkt, darunter auch Forscher und Forscherinnen aus Frankreich, Österreich, der Schweiz, aus den USA, Israel und Rußland. Die Biographien sind 
alphabetisch geordnet, der Leser kann sich aber auch in einer chronologischen Übersicht einen ersten Eindruck über die Auswahl verschaffen. Über die Auswahl kann man streiten, und jeder, der ein Lexikon macht, weiß auch, daß nicht alle Blütenträume reifen. Die Herausgeberin der auf 56 Bände und 26300 Artikel angewachsenen "Allgemeinen Deutschen Biographie“, die Bayerische Akademie der Wissenschaften, ließ in der Vorrede zum letzten, 1912 erschienenen Band, lakonisch verlauten: "Von den 1850 Verfassern der einzelnen Lebensbeschreibungen ist nun, im Laufe von fast vierzig Jahren, weitaus die Mehrzahl bereits selbst dahingeschieden*. Soweit konnten und wollten es die Herausgeber des vorliegenden Lexikons "Demokratische Wege" nicht kommen lassen.

Die Herausgeber danken allen Personen und Institutionen, die zum Gelingen der Publikation beigetragen haben. In erster Linie danken sie Jan Philipp Reemtsma, der das Unternehmen ermöglichte, an den konzeptionellen Gesprächen beteiligt war und den Fortgang begleitete.

Manfred Asendorf

Rolf von Bockel 